\title{
HD144941: The most extreme helium-strong star
}

\author{
N. Przybilla ${ }^{1}$, L. Fossati ${ }^{2}$, and C.S. Jeffery ${ }^{3}$ \\ ${ }^{1}$ Institut für Astro- und Teilchenphysik, Universität Innsbruck, Technikerstr. 25/8, 6020 Innsbruck, Austria \\ e-mail: norbert.przybilla@uibk.ac.at \\ 2 Space Research Institute, Austrian Academy of Sciences, Schmiedlstr. 6, 8042 Graz, Austria \\ 3 Armagh Observatory and Planetarium, College Hill, Armagh BT61 9DG, N. Ireland \\ Received ; accepted
}

\begin{abstract}
Since its discovery about 50 years ago, HD144941 has generally been classified as a peculiar member of the extreme helium (EHe) supergiant stars, a very rare class of low-mass hydrogen-deficient stars. We report the detection of a strong longitudinal magnetic field based on spectropolarimetry with FORS2 on the ESO VLT with surface-averaged longitudinal field strengths as large as -9 kG. This is further constrained by the detection of Zeeman splitting of spectral lines to a field strength of at least $15 \mathrm{kG}$, explaining the recent finding of surface spots for this star. The quantitative analysis of the stellar atmosphere based on a hybrid non-local thermodynamic equilibrium approach and new optical spectra yields an effective temperature of $22000 \pm 500 \mathrm{~K}$, a logarithmic surface gravity of $4.20 \pm 0.10$, and a surface helium fraction of $0.950 \pm 0.002$ by number. While the metal abundances are about a factor of 10 sub-solar in absolute number, the metal-to-hydrogen ratios are typical of massive early-type stars, indicating that helium fallback in a weak, fractionated stellar wind in the presence of a magnetic field took place - the canonical mechanism for the formation of the helium-strong phenomenon. Both the spectroscopic and the Gaia EDR3 parallax imply HD144941 to be a luminous massive star. Kinematically, we argue that HD144941 has reached its high Galactic latitude as a runaway star. We conclude that instead of being a comparatively high-gravity low-mass EHe star, HD144941 is by far the most extreme member of the magnetic massive helium-strong stars, with almost all atmospheric hydrogen substituted by helium.
\end{abstract}

Key words. Stars: abundances - Stars: early-type - Stars: fundamental parameters - Stars: magnetic field - Stars: massive

\section{Introduction}

The helium-peculiar nature of HD144941 was first noticed by MacConnell et al. (1970). An early quantitative analysis by Hunger \& Kaufmann (1973) confirmed it to be a member of an extreme subclass of B-type hydrogen-deficient stars with low mass and a comparatively large radius, the so-called extreme helium (EHe) stars (see Jeffery 2008 for a review). The first spectral analysis of HD144941 using a modern model atmosphere code by Harrison \& Jeffery (1997) and Jeffery \& Harrison (1997) based on the assumption of local thermodynamic equilibrium (LTE) established its peculiarity among the EHe stars. It found a comparatively high gravity, an unusually high hydrogen abundance of $\sim 5 \%$, a metallicity of 1.6 dex below solar, and abundances not showing the products of any nuclear reactions other than hydrogen burning, in contrast to the presence of CNO- and $3 \alpha$-process ashes in most other EHe stars. Yet, the star appeared to show properties much closer to those of the EHe stars (with helium contents of $\sim 99 \%$ ) than to those of any other helium-rich stars, in particular the magnetic massive helium-strong main sequence stars, which show helium contents typically in the 30 to $50 \%$ range (e.g. Smith 1996; Cidale et al. 2007). Later, non-LTE analyses confirmed the earlier findings (Przybilla et al. 2005, 2006; Pandey \& Lambert 2017). The merger of two helium white dwarfs in a binary was identified as a viable formation mechanism (Saio \& Jeffery 2000). The recent detection of rotational modulation in a light curve of HD144941 (Jeffery \& Ramsay 2018) measured by the $\mathrm{K} 2$ mission points to the existence of surface spots, which in turn are indicative for the presence of a magnetic field in an early-type star. In the following, we discuss the first spectropolarimetric observations of the star and perform a quantitative spectral reanalysis, which show that HD144941 is indeed a very different type of star than supposed to date.

\section{Observations}

Our target star HD144941 was observed in service mode on a total of nine nights in March, June, and July 2021 using the FOcal Reducer and low dispersion Spectrograph 2 (FORS2) lowresolution spectropolarimeter (Appenzeller et al. 1998) attached to the European Southern Observatory (ESO) Very Large Telescope (VLT) Unit Telescope 1 (UT1) at Cerro Paranal, Chile. On the night of July 1, 2021, we obtained two sets of observations separated by a few hours. The data were obtained with a slit width of $0.4^{\prime \prime}$ and the grism $600 \mathrm{~B}$. This setting yielded a resolving power $R \approx 1700$ and a wavelength coverage from 3250 to $6215 \AA$. On each night of observation, we obtained several spectra with an exposure time of 30 seconds each. We obtained a first set of spectra with the quarter wave plate at $-45^{\circ}$, then twice as many at $+45^{\circ}$, and then again exposures at $-45^{\circ}$, to finally obtain the same amount of spectra for each of the two angle positions. The observing log and Stokes $I$ signal-to-noise ratios $(\mathrm{S} / \mathrm{N})$ per pixel calculated around $4950 \AA$ are listed in Table 1 . The data were reduced using custom-made tools described in detail by Fossati et al. (2015), which are based on the recommendations of Bagnulo et al. (2012).

An optical spectrum with $R=48000$ was obtained on April 8, 2006 using the Fibre-fed Optical Echelle Spectrograph (FEROS, Kaufer et al. 1999) on the ESO-Max-PlanckGesellschaft (MPG) 2.2m telescope at La Silla, Chile. The 

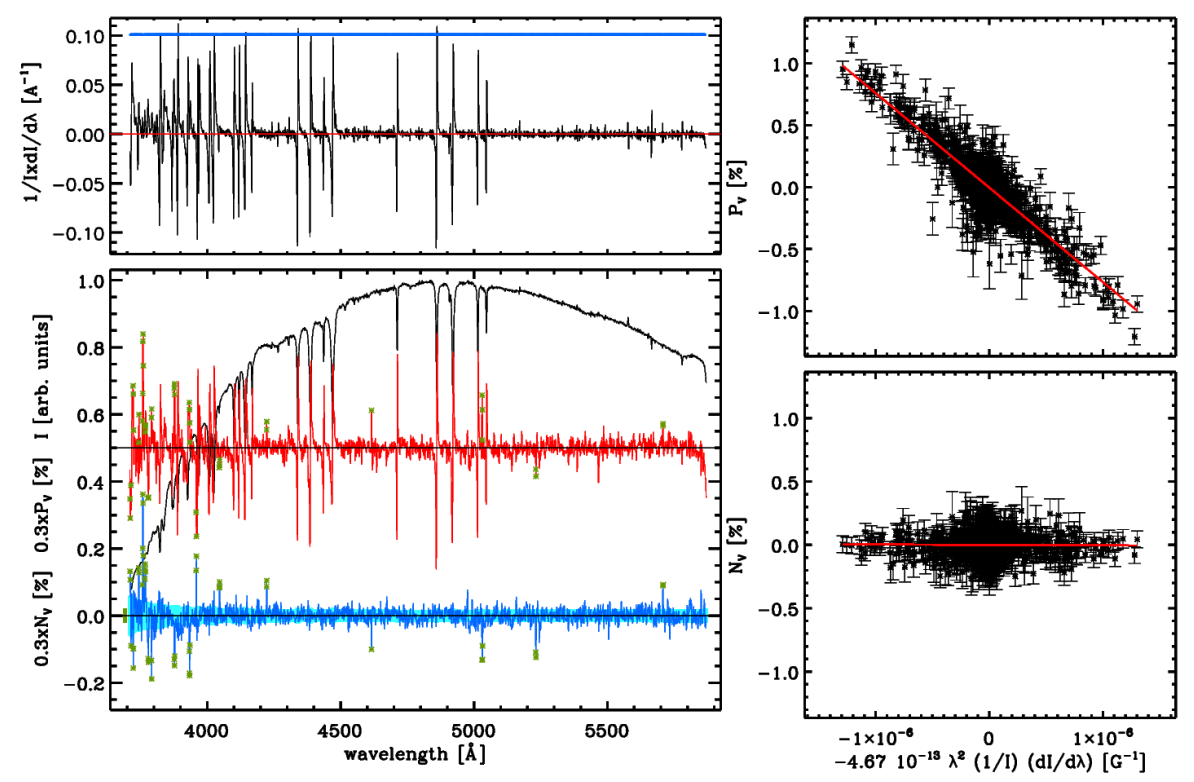

Fig. 1. Overview of FORS2 analysis and results from the observations of HD144941 collected on March 15, 2021 considering the full spectrum. Top left panel: Derivative of Stokes $I$. The thick blue line on top of the panel indicates the region used to compute $\left\langle\mathrm{B}_{z}\right\rangle$. Bottom left panel: From top to bottom, the panel shows the Stokes $I$ spectrum (black) arbitrarily normalised to the highest value, the Stokes $V$ spectrum (in \%; red) rigidly shifted upwards by $0.5 \%$ for visualisation reasons, and the spectrum of the $N$ parameter (in \%; blue). The green asterisks mark the points that were removed by the sigma-clipping algorithm. The pale blue strip drawn on top of the $N$ profile shows the uncertainty associated with each spectral point. The thick green bar on the left side of the spectrum of the $N$ parameter shows the standard deviation of the $N$ profile. Top right panel: Linear fit from which $\left\langle\mathrm{B}_{z}\right\rangle$ is determined. The red solid line shows the best fit giving $\left\langle\mathrm{B}_{z}\right\rangle=-7626 \pm 86 \mathrm{G}$. Bottom right panel: Same as the top right panel, but for the $N$ profile (i.e. $\left\langle\mathrm{N}_{z}\right\rangle$ ), from which we obtain $\left\langle\mathrm{N}_{z}\right\rangle=-32 \pm 71 \mathrm{G}$.

Table 1. FORS2 Observing log and average longitudinal magnetic field $\left(\left\langle B_{z}\right\rangle\right)$ values.

\begin{tabular}{lc|ccc|cr|rr}
\hline \hline $\begin{array}{l}\text { Date } \\
\text { YYYY-MM-DD }\end{array}$ & $\begin{array}{c}\text { JD- } \\
2450000\end{array}$ & $\begin{array}{c}\text { No. of } \\
\text { frame }\end{array}$ & $\begin{array}{c}\text { Exposure } \\
\text { time }(\mathrm{s})\end{array}$ & $\begin{array}{c}\text { S/N at } \\
4950 \AA\end{array}$ & \multicolumn{2}{|c|}{$\left\langle\mathrm{B}_{z}\right\rangle(\mathrm{G})$} & $\left\langle\mathrm{N}_{z}\right\rangle(\mathrm{G})$ & \multicolumn{2}{|c|}{$\left\langle\mathrm{B}_{z}\right\rangle(\mathrm{G})$} & $\left\langle\mathrm{N}_{z}\right\rangle(\mathrm{G})$ \\
\hline $2021-03-15$ & 9288.74493 & 40 & 30 & 1741 & $-9004 \pm 170$ & $-129 \pm 137$ & $-7626 \pm 86$ & $-32 \pm 71$ \\
$2021-03-24$ & 9297.70603 & 40 & 30 & 1975 & $-8146 \pm 159$ & $16 \pm 135$ & $-7068 \pm 92$ & $-117 \pm 73$ \\
$2021-06-10$ & 9376.49805 & 40 & 30 & 1307 & $-7377 \pm 266$ & $278 \pm 219$ & $-6718 \pm 134$ & $154 \pm 114$ \\
$2021-06-12$ & 9377.78924 & 40 & 30 & 1886 & $-7963 \pm 189$ & $-28 \pm 165$ & $-7043 \pm 85$ & $97 \pm 73$ \\
$2021-07-01 \mathrm{a}$ & 9396.15904 & 40 & 30 & 2156 & $-7549 \pm 140$ & $85 \pm 117$ & $-6538 \pm 70$ & $16 \pm 55$ \\
$2021-07-01 b$ & 9396.21277 & 80 & 30 & 2761 & $-7283 \pm 126$ & $-48 \pm 100$ & $-6422 \pm 67$ & $-27 \pm 51$ \\
$2021-07-08$ & 9403.13370 & 36 & 30 & 1738 & $-7823 \pm 164$ & $-238 \pm 116$ & $-6872 \pm 81$ & $-80 \pm 68$ \\
$2021-07-11$ & 9406.12985 & 36 & 30 & 1539 & $-8375 \pm 222$ & $-799 \pm 202$ & $-6820 \pm 105$ & $-362 \pm 86$ \\
$2021-07-14$ & 9409.14539 & 18 & 30 & 782 & $-8382 \pm 424$ & $690 \pm 304$ & $-6721 \pm 208$ & $128 \pm 154$ \\
$2021-07-18$ & 9413.10564 & 36 & 30 & 1490 & $-8914 \pm 194$ & $-170 \pm 171$ & $-7806 \pm 103$ & $-55 \pm 87$ \\
\hline
\end{tabular}

Notes. Julian date listed in the second column is that at the beginning of the exposure sequence. Columns three and four give the number of frames obtained during each night of observation and the exposure time of each frame, respectively. Column five lists the $\mathrm{S} / \mathrm{N}$ per pixel of the intensity spectrum (Stokes $I$ ) calculated at $\approx 4950 \AA$ Aver a wavelength range of $100 \AA$. Columns six and seven list the $\left\langle\mathrm{B}_{z}\right\rangle$ and $\left\langle\mathrm{N}_{z}\right\rangle$ values obtained employing the hydrogen lines from the Stokes $V$ and $N$ parameter spectra, respectively. Columns eight and nine are the same as columns six and seven, but employing the full spectrum.

$3000 \mathrm{~s}$ exposure yielded a peak $\mathrm{S} / \mathrm{N}$ of about 200. The data were complemented by a $780 \mathrm{~s}$ exposure with the Ultraviolet and Visual Echelle Spectrograph (UVES, Dekker et al. 2000) on the ESO-VLT UT2 on April 10, 2006, covering the optical-UV range at $R \approx 41000$ and reaching a peak-S/N$\approx 170$. The combined wavelength range at $\mathrm{S} / \mathrm{N}$ high enough for a quality analysis covers $\sim 3200$ to $8000 \AA$. The data reduction was performed in analogy to Kupfer et al. (2017). We want to emphasise that an additional spectrum of the DC white dwarf WD 1917-07 was observed with UVES to trace the spectral response function, facilitating the normalisation of the target spectra in an objective way (see Koester et al. (2001) for details of the method). In analogy, the spectrum of the (well-modelled) sdB star HD188112 was employed for normalisation of the FEROS data.

Low-dispersion and large-aperture spectrophotometry from the International Ultraviolet Exporer (IUE) was employed to constrain the spectral energy distribution (SED) of HD144941 (data sets SWP07696 and LWR06703). These were supplemented by photometry in the Johnson $U B V$-bands from Slawson et al. (1992), in the Two Micron All Sky Survey (2MASS) $J H K$-bands from Skrutskie et al. (2006), and in the Wide-field Infrared Survey Explorer (WISE) bands from Cutri et al. (2021).

\section{Magnetic field detection}

We reduced the FORS2 spectropolarimetric observations employing the pipeline described by Fossati et al. (2015), which is based on that of Bagnulo et al. (2012, 2015). Furthermore, the pipeline implements the set of tests recommended by Bagnulo et al. (2013) to aid the identification of genuine magnetic field detections. The surface-averaged longitudinal magnetic field values obtained from the analysis of the FORS2 observations and conducted on both the Stokes $V$ spectrum and null $(N)$ profile are listed in Table 1. We show in Fig. 1 the output of the analysis performed on the observations obtained on March 15, 2021, and considering the whole spectrum. The strong magnetic field detection is indicated by the significant slope shown in the top right panel, while the lack of a significant slope in the bottom right panel indicates to the first order that the magnetic field detection is genuine (see Bagnulo et al. 2013 for a thorough discussion).

Table 1 shows that we obtained a significant magnetic field detection $(\gg 10 \sigma)$ from each night of observation, with a peak strength of $\left\langle\mathrm{B}_{z}\right\rangle \approx-9 \mathrm{kG}$ obtained from the data collected on March 15 and July 18, 2021, and considering the hydrogen lines. We obtained a significant magnetic field detection employing the 


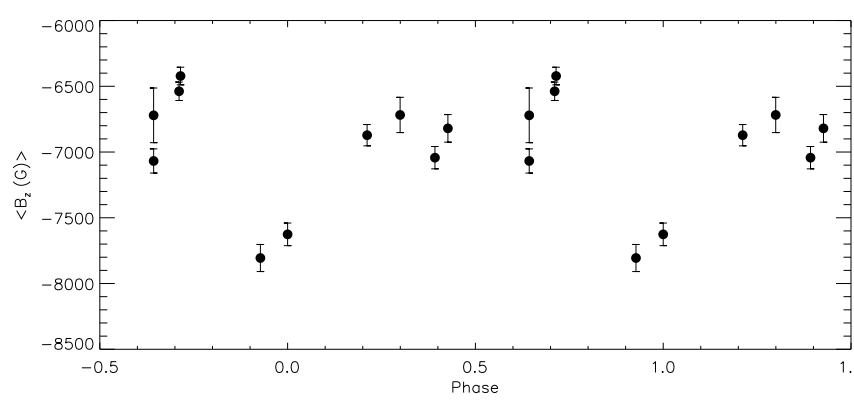

Fig. 2. Average longitudinal magnetic field variation of HD144941 from measurements of the whole spectrum, phased by the $13.93 \mathrm{~d}$ period derived by Jeffery \& Ramsay (2018) from the K2 light curve. The zero point is given by our first measurement, coinciding close to the maximum of the magnetic field strength. We note the repetition of the data below phase 0 and above phase 1 for visualisation purposes.

hydrogen lines, or the metal lines (not shown here), or the whole spectrum. The $\left\langle\mathrm{B}_{z}\right\rangle$ values obtained from the hydrogen lines are on average $1-1.4 \mathrm{kG}$ stronger than those obtained from the metal lines or the whole spectrum. For strongly magnetic stars, such discrepancies are common and can be ascribed, for example, to the (partial) breaking of the weak-field approximation on which the magnetic field measurement is based (Angel \& Landstreet 1970; Borra \& Landstreet 1980; Bagnulo et al. 2002; Landstreet et al. 2014; Fossati et al. 2015).

Instead, the variation of $1-2 \mathrm{kG}$ in the $\left\langle\mathrm{B}_{z}\right\rangle$ values obtained from the March, June, and July data (see Table 1 and Fig. 2) can be ascribed to rotational modulation. Jeffery \& Ramsay (2018) found a highly structured singly periodic signal in K2 observations, with a fundamental period of $13.9 \pm 0.2 \mathrm{~d}$ and multiple harmonics. We adopted the value of $13.93 \mathrm{~d}$ (their Fig. 3) to phase the $\left\langle\mathrm{B}_{z}\right\rangle$ values from analysis of the whole spectrum in Fig. 2. While our data are insufficient to improve on the period derived from the K2 light curve, they are consistent with it. As the polarity of the magnetic field does not change over the full period, a viewing geometry not too far from pole-on can be deduced, with the magnetic field also coarsely aligned with the rotation axis. We note that the structured light curve is indicative of a magnetic field configuration more complex than a simple dipole, but more observations are required to constrain the magnetic field geometry.

While the presence of a magnetic field (and rotational modulation) has not been found for any other EHe star, magnetic fields are crucial for the development of the He-strong phenomenon among massive main-sequence B-type stars. This means that the notion of HD144941 being an EHe star should be reconsidered.

\section{Model atmosphere analysis}

For the quantitative analysis of the spectra we followed a hybrid non-LTE approach (Nieva \& Przybilla 2007; Przybilla et al. 2011) that we used before to study both EHe stars (Kupfer et al. 2017) and helium-strong stars (e.g. Przybilla et al. 2016; Castro et al. 2017). In brief, LTE model atmospheres as computed with the AtLas 12 code (Kurucz 1996) were used as basis for subsequent non-LTE line-formation calculations with DeTAIL and Surface (Giddings 1981; Butler \& Giddings 1985 both updated and extended by K. Butler). The former code solves the coupled statistical equilibrium and radiative transfer equations, while the latter computes the emergent synthetic spectrum based on the resulting non-LTE population numbers using refined line-
Table 2. Parameters and elemental abundances of HD144941.

\begin{tabular}{|c|c|c|c|}
\hline \multicolumn{4}{|c|}{ Atmospheric parameters: } \\
\hline$T_{\text {eff }}$ & $22000 \pm 500 \mathrm{~K}$ & $\xi$ & $2 \pm 1 \mathrm{~km} \mathrm{~s}^{-1}$ \\
\hline $\log g / \mathrm{cm} \mathrm{s}^{-2}$ & $4.20 \pm 0.10$ & $v \sin i$ & $7 \pm 5 \mathrm{~km} \mathrm{~s}^{-1}$ \\
\hline$y$ (by number) & $0.950 \pm 0.002$ & $\zeta$ & $25 / 15 \pm 5 \mathrm{~km} \mathrm{~s}^{-1 a}$ \\
\hline \multicolumn{4}{|c|}{ Non-LTE abundances $\log n_{X}$, normalised to $\sum n_{X}=1$ : } \\
\hline $\mathrm{H}$ & $-1.30 \pm 0.02(4)$ & $\mathrm{Mg}$ & $-5.52(1)$ \\
\hline $\mathrm{C}$ & $-4.63 \pm 0.12(7)$ & $\mathrm{Al}$ & $-6.64 \pm 0.06(4)$ \\
\hline $\mathrm{N}$ & $-5.06 \pm 0.09(15)$ & $\mathrm{Si}$ & $-5.48 \pm 0.11(15)$ \\
\hline $\mathrm{O}$ & $-4.26 \pm 0.07(22)$ & $\mathrm{S}$ & $-5.61 \pm 0.10(5)$ \\
\hline $\mathrm{Ne}$ & $-4.67 \pm 0.04(2)$ & $\mathrm{Fe}$ & $-5.41 \pm 0.13(5)$ \\
\hline \multicolumn{4}{|c|}{ Photometric data: } \\
\hline$V$ & $10.141 \pm 0.008 \mathrm{mag}^{b}$ & $R_{V}$ & $3.8 \pm 0.1$ \\
\hline$B-V$ & $0.034 \pm 0.007 \mathrm{mag}^{b}$ & $M_{V}$ & $-1.85^{+0.10} \mathrm{mag}$ \\
\hline$E(B-V)$ & $0.27 \pm 0.01 \mathrm{mag}$ & $M_{\mathrm{bol}}$ & $-3.94 \pm 0.10 \mathrm{mag}$ \\
\hline \multicolumn{4}{|c|}{ Fundamental parameters: } \\
\hline$M / M_{\odot}$ & $8.1 \pm 0.3$ & $\tau$ & $11.0_{-78}^{+6.4} \mathrm{Myr}$ \\
\hline$R / R_{\odot}$ & $3.8 \pm 0.2$ & $\tau / \tau_{\mathrm{MS}}$ & $0.29_{-0.21}^{+0.20}$ \\
\hline $\log L / L_{\odot}$ & $3.47 \pm 0.04$ & $P_{\text {rot }}$ & $13.9 \pm 0.2 \mathrm{~d}^{c}$ \\
\hline \multicolumn{4}{|c|}{ Kinematic data: } \\
\hline$\pi_{\mathrm{EDR} 3}$ & $0.6714 \pm 0.0282 \mathrm{mas}^{d}$ & & $-43.7 \pm 0.5 \mathrm{~km} \mathrm{~s}^{-1}$ \\
\hline & $-6.834 \pm 0.034 \mathrm{mas} \mathrm{yr}^{-1 d}$ & $d_{\text {Gaia }}$ & $1556_{-66}^{+71} \mathrm{pc}$ \\
\hline & $8.023 \pm 0.025 \mathrm{mas} \mathrm{yr}^{-1 d}$ & $d_{\text {spec }}$ & $1582 \pm 208 \mathrm{pc}$ \\
\hline
\end{tabular}

Notes. $1 \sigma$-uncertainties are given. For abundances, these are from the line-to-line scatter; systematic errors amount to an additional $\sim 0.1$ dex. (a) with and without considering Zeeman splitting (b) Slawson et al. (1992) $^{(c)}$ Jeffery \& Ramsay (2018) ${ }^{(d)}$ Gaia Collaboration et al. (2016, 2021)

broadening theories. Here, detailed broadening tables of Tremblay \& Bergeron (2009) were used with the model atoms for $\mathrm{H}$ (Przybilla \& Butler 2004), and those of Beauchamp et al. (1997) were used for He I (Przybilla 2005). We adopted model atoms for the metals according to Table 2 of Przybilla et al. (2016).

The atmospheric parameters, which are effective temperature $T_{\text {eff }}$, surface gravity $g$, helium abundance (by number) $y$, microturbulence $\xi$, macroturbulence $\zeta$, projected rotational velocity $v \sin i$ as well as abundances $\log n_{X}$ (number fractions, normalised to $\sum n_{X}=1$ ) of chemical species $X$ were determined from line-profile fits. Important diagnostics for the analysis were the Stark-broadened hydrogen Balmer and $\mathrm{He}_{\mathrm{I}}$ lines, and the ionisation balances of $\mathrm{Si}$ II/III and $\mathrm{S}$ II/III were established simultaneously (i.e. abundances from different ionisation stages were brought to match). Independence of the abundances from the strength of the analysed lines was used as criterion to fix $\xi$. For a detailed description of the iterative procedure, we invite the reader to consult Nieva \& Przybilla (2012).

The derived atmospheric parameters and elemental abundances are summarised in Table 2 . The quality of the atmospheric parameter solution can be assessed best by the overall excellent match between the observed and the final model spectrum. This is demonstrated in Fig. 3 for all hydrogen Balmer and $\mathrm{He}_{\mathrm{I}}$ lines between 3900 and $7100 \AA$, plus two lines in the optical-UV, He I $\lambda \lambda 3187.74 \AA$ and $3354.56 \AA$. He II $\lambda 4686 \AA$ also imposes a tight upper limit on $T_{\text {eff }}$ due to its extreme weakness in the spectrum. Small increases in $T_{\text {eff }}$ would lead to a more pronounced line. Minor deviations between observations and the model become apparent only for the lines at $\lambda \gtrsim 5000 \AA$, such that the observed lines are broader than the model, increasingly so with wavelength; this point becomes significant below. Additionally, an overall good match of the metal lines is obtained, reproducing the observed profiles nicely by the model 

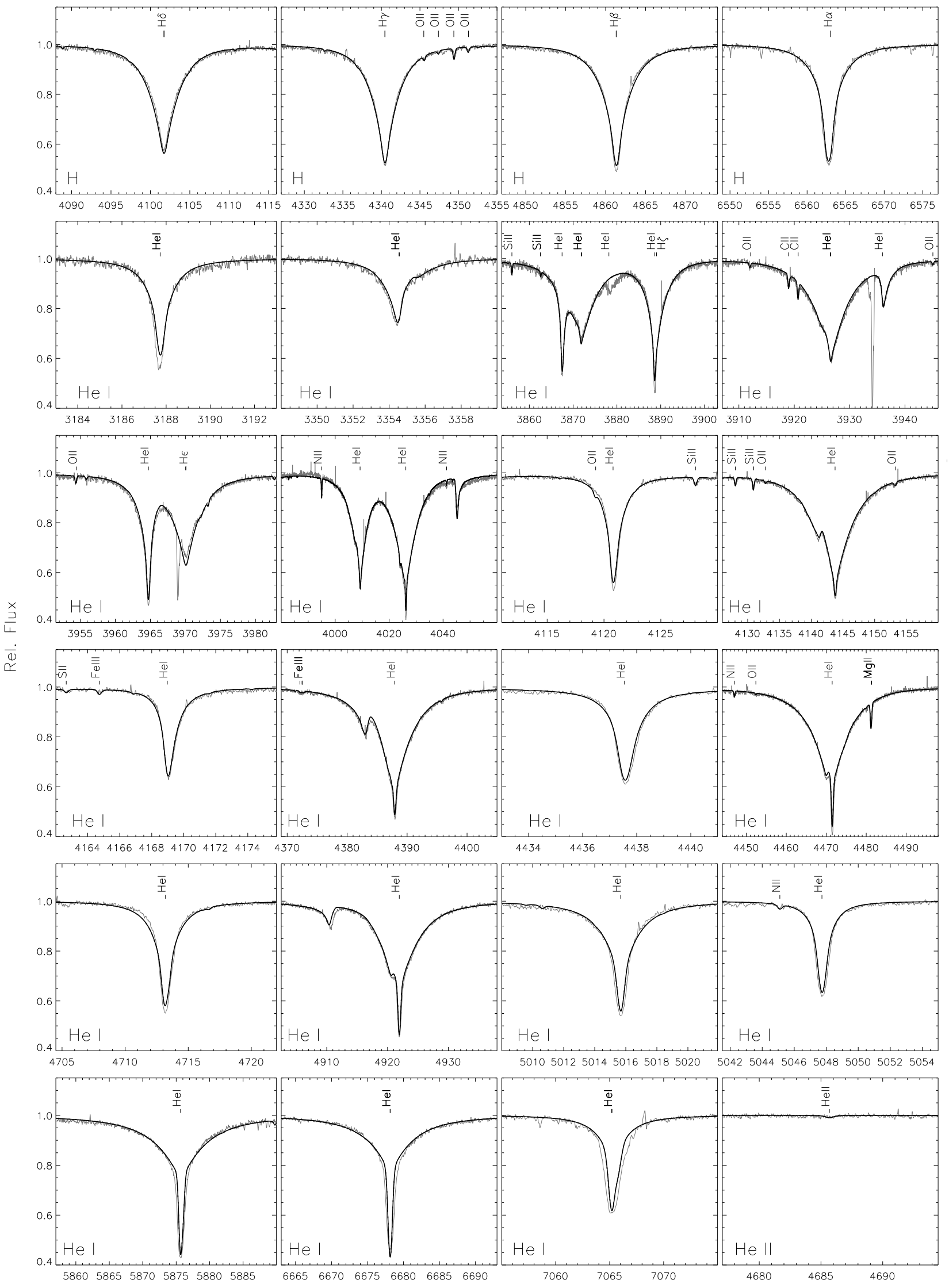

$\lambda(\AA)$

Fig. 3. Comparison of final model spectrum (black lines) with observed hydrogen and helium lines in HD144941 (grey lines). 


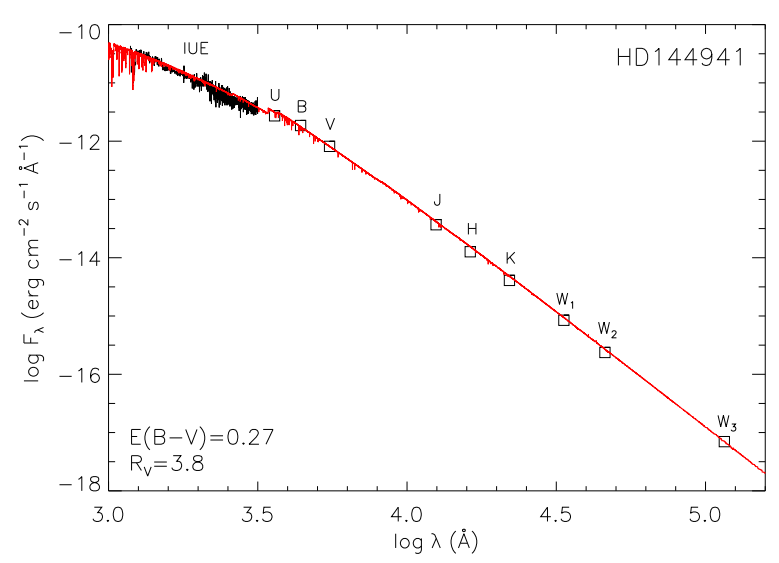

Fig. 4. Comparison of de-reddened spectral energy distribution of HD144941 (black line, squares) with the model non-LTE flux (red).

finally adopted, establishing ionisation equilibria of Si II/III and $\mathrm{S}$ II/III. Some of the stronger lines show deviations from a rotational/macroturbulent profile in their cores, just above the noise level - this discussion will also be resumed later. Noteworthy in the comparison with previous work is the large discrepancy with respect to the $\log g=3.45 \pm 0.15$ determined by Pandey \& Lambert (2017) and our much lower microturbulence $\left(2 \mathrm{~km} \mathrm{~s}^{-1}\right.$ vs. $10 \mathrm{~km} \mathrm{~s}^{-1}$ in the literature). One may speculate about continuum normalisation as a reason for the former because of the much larger widths of the $\mathrm{H}$ and $\mathrm{He}$ lines than in typical EHe stars; we invite the reader to compare the widths of lines in Figs. 7 and 9 of Pandey \& Lambert (2017) with our data in Fig. 3.

Constraints on $T_{\text {eff }}$ are also obtained from the fit of the nonLTE model fluxes to the observed SED of the star (see Fig. 4 for the match achieved from the UV to the thermal IR). The observed fluxes were de-reddened using an interstellar reddening law according to Cardelli et al. (1989), fitting both the colour excess $E(B-V)$ and the ratio of total-to-selective extinction $R_{V}=A_{V} / E(B-V)^{1}$. The resulting extinction and the Gaia distance provided the absolute visual magnitude $M_{V}$ of the star, and the application of the computed bolometric correction from the stellar atmosphere model provided the bolometric magnitude $M_{\text {bol }}$. The observed $V$ and $B-V$ data for HD144941 and the deduced photometric data are summarised in Table 2.

Detailed abundances from our line-by-line analysis are summarised in Table A.1. For each spectral line, the wavelength $\lambda$ is stated, as well as the excitation energy $\chi$ of the lower level of the transition, the adopted oscillator strength $\log g f$, an accuracy flag for it and its source, and the non-LTE abundance value $n_{X}$. Average values are given in Table 2, with the number of analysed lines indicated in brackets. Our metal abundances are about a factor four higher than in previous determinations, but they are still rather low, at around a value of about 1/10 solar (see the upper panel of Fig. 5). Carbon is normal, in contrast to the typical EHe stars where the carbon number fraction is of the order of $1 \%$ (i.e. $~ 50 \times$ solar) due to admixture of $3 \alpha$-process ash. Nitrogen is slightly enriched relatively to carbon and oxygen, which may be a sign for mixing with small amounts of CN-cycled matter. Some $\alpha$-elements like neon or sulphur are overabundant with respect to

\footnotetext{
1 A comparatively high reddening value is found for the star's position $17.8^{\circ}$ above the Galactic plane, and an anomalous reddening law. Most of the effect should stem from the well-known foreground dust lanes in the Scorpius region.
}

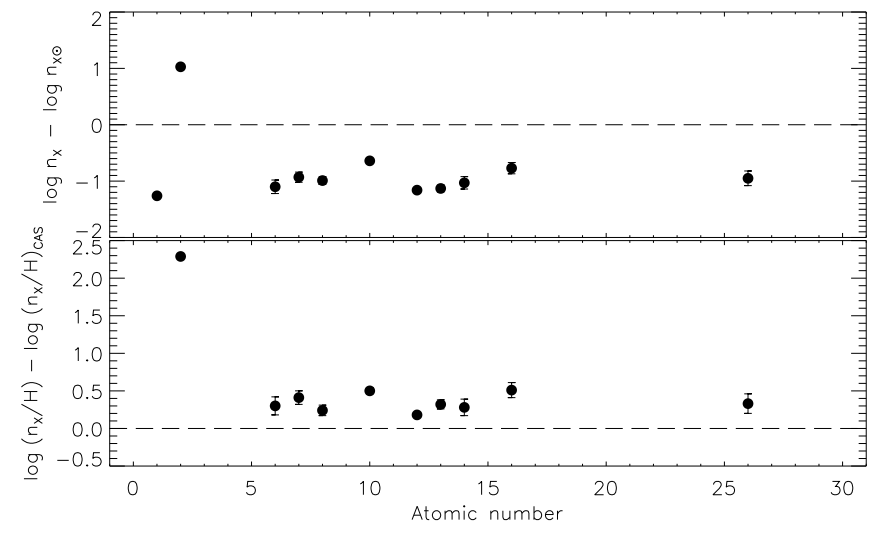

Fig. 5. Upper panel: Elemental abundances $\log n_{X}$ of HD144941 (dots) compared to solar values (Asplund et al. 2009). Lower panel: Elemental abundances $\log \left(n_{X} / \mathrm{H}\right)$ relative to cosmic abundances (Nieva \& Przybilla 2012; Przybilla et al. 2013). The dashed lines mark the identity.

iron, which has been interpreted in the past as $\alpha$-enhancement as typically found in thick-disc or halo stars.

However, a change of perspective brings new insight. If the metal abundances are normalised to the hydrogen abundance and compared to abundances found for young stars in the solar neighbourhood (the cosmic abundance standard, CAS; Nieva \& Przybilla 2012; Przybilla et al. 2013), the values appear super-solar by about a factor of two (see the lower panel of Fig. 5). This is what one would expect to find for a young star from the inner Milky Way. The canonical mechanism for producing the helium enrichment of the atmospheric layers of He-strong stars via a weak, fractionated stellar wind in the presence of a magnetic field (Hunger \& Groote 1999) could indeed lead to the observed pattern in absolute numbers. The metal-line-driven wind drags the bulk plasma with it, but while Coulomb coupling efficiently accelerates the ionised hydrogen, the neutral helium falls back to the atmosphere. Over time, helium accumulates ${ }^{2}$ and reduces the absolute abundances of the other chemical constituents. The star HD144941 is special insofar as the process occurred to a much more pronounced extent than in any other He-strong star known.

\section{Kinematic analysis}

We employed the approach of Pauli et al. (2006) for the computation of the orbit and the kinematic parameters using the code of Odenkirchen \& Brosche (1992). The Galactic potential of Allen $\&$ Santillan (1991) was used. Parallax $\pi_{\mathrm{EDR} 3}$ and proper motion data $\mu_{\alpha}$ and $\mu_{\delta}$ from Gaia Early Data Release 3 (EDR3 Gaia Collaboration et al. 2016, 2021) were utilised. The barycentric radial velocity $v_{\text {rad }}$ was measured from the FEROS spectrum. The Gaiabased distance $d_{\text {Gaia }}$ was determined adopting a Gaia EDR3 parallax zero-point offset by Ren et al. (2021), and the spectroscopic distance following Nieva \& Przybilla (2012). The kinematic data and distances are also collected in Table 2.

Based on the Gaia EDR3 parallax and proper motions the geometric form of the orbit of HD144941 appears to be thickdisc-like both in the Galactic plane and the meridional projection $(X, Y$, and $Z$ are Cartesian Galactic coordinates with the Galactic centre at the origin, and $\rho$ is the Galactocentric radius; see the 2 This produces a negative $\mu$-gradient ( $\mu$ being the mean molecular
weight) which is unstable against turbulent motions that might trans-
port material of pristine composition to the surface, thus removing the chemical peculiarity. However, a magnetic field suppresses turbulence. 

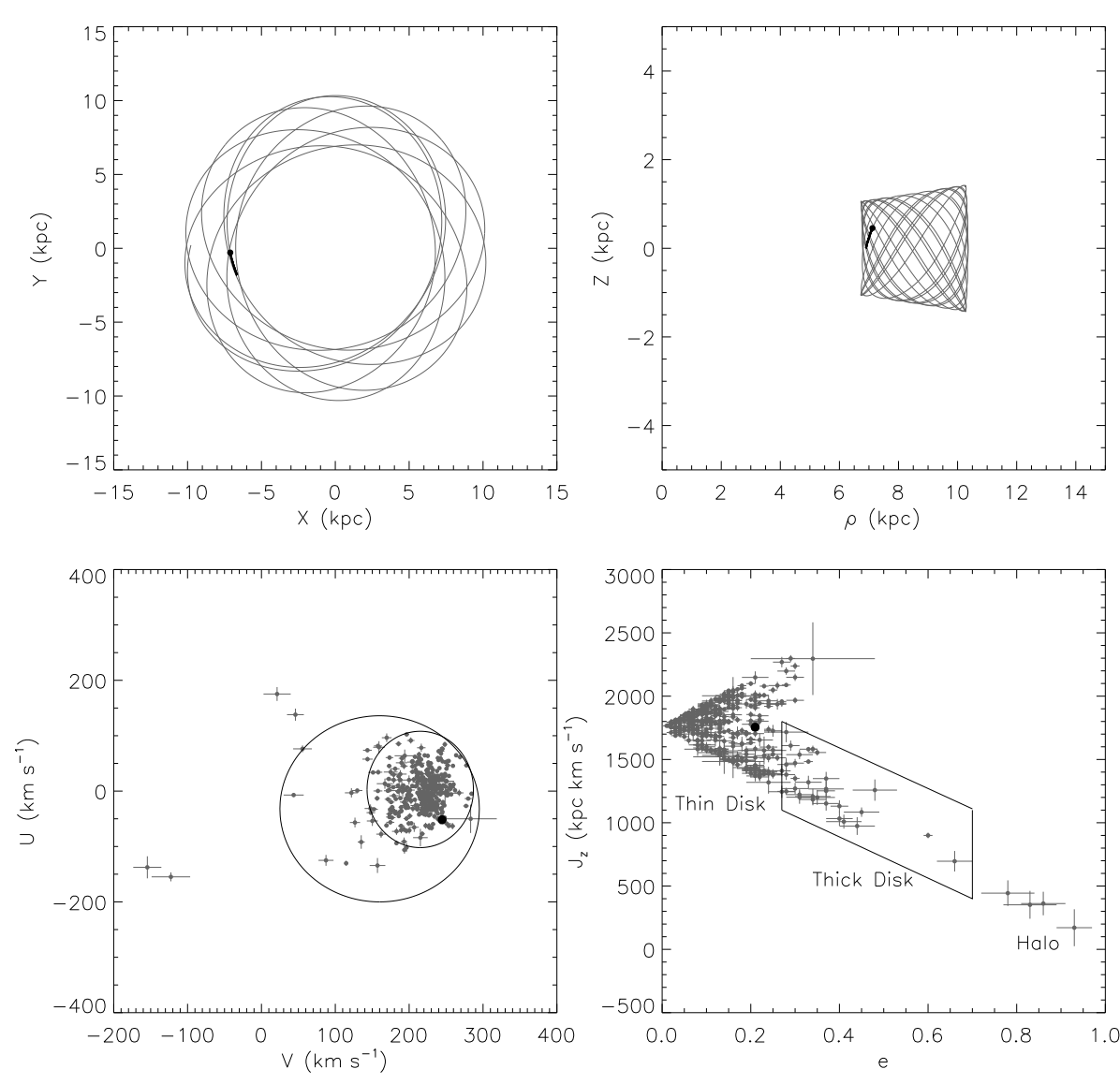

Fig. 6. Kinematics of HD144941 (black dots for present values) with respect to the kinematic data for the white dwarf sample (grey dots with error bars) of Pauli et al. (2006). Upper left panel: Orbital motion of HD144941 in the Galactic plane. Upper right panel: Meridional orbit. Backward integration by about $6.2 \mathrm{Myr}$ to the Galactic equatorial plane crossing is indicated by the full black line, integration from the present to $+2 \mathrm{Gyr}$ by the grey line. Lower left panel: $V-U$ velocity plot. The black ellipses render the $3 \sigma$ thin (inner) and thick disc (outer) contours as specified by Pauli et al. (2006). Lower right panel: $e-J_{z}$ diagram. The solid box marks the thick-disc region as specified by Pauli et al. (2006), separating the thin disc and the halo region. grey curve in both upper panels of Fig. 6, which was calculated 2 Gyr into the future). However, the velocity components of the star in the radial and Galactic rotation direction $(U, V)$ do not match those of typical thick-disc stars (according to the criteria employed by Pauli et al. 2006), and neither do the eccentricity and $Z$-component of the angular momentum $J_{Z}$ (lower panels of Fig. 6), which are thin-disc-like. This contradiction can be resolved if HD144941 is a runaway star, ejected about 6.2 Myr ago from the Sagittarius spiral arm in the Galactic plane (black lines), either by dynamical ejection or by a supernova exploding in a binary system. During its remaining lifetime, HD144941 will climb in Galactic latitude until presumably exploding in a supernova about $1.1 \mathrm{kpc}$ above the Galactic plane, possibly leaving a magnetar as remnant.

\section{HD144941 in the evolutionary context}

Finally, fundamental stellar parameters such as mass $M$, radius $R$, luminosity $L$, age $\tau$, and ratio of age-to-main-sequence lifetime $\tau / \tau_{\text {MS }}$ were determined following Nieva \& Przybilla (2014). The Gaia EDR3 parallax implies beyond any doubt that HD144941 is a luminous massive star and not of low mass, despite the similar $L / M$-ratio found for the object if it were an EHe star. As a consequence, evolution tracks like those of the Geneva group (Ekström et al. 2012) are appropriate for constraining the stellar mass (and age) from a Kiel diagram to $8.1 \pm 0.3 M_{\odot}$, see Fig. 7.

When compared to the positions of other He-strong stars as collected from the literature (e.g. Zboril et al. 1997; Leone et al. 1997; Hunger \& Groote 1999; Cidale et al. 2007) (see also Ghazaryan et al. (2019) for a recent compilation ), HD144941 does not appear to be distinguished at first glance (see Fig. 7). However, the two stars HD58260 and HD64740 that were analysed in all four literature studies yield $T_{\text {eff-values different by }}$ many thousands of Kelvin, and $\log g$-values that span the mainsequence band, for the same star. Apparently, systematic uncertainties of the different approaches can be much larger than indicated by the respective error bars. Moreover, about one-third of the literature values locate the stars below the zero-age main sequence (ZAMS), a characteristic that is unexpected for objects of chemically-normal composition except for their atmospheric layers. We conclude from this that a meaningful comparison may not be feasible by considering these previous studies obviously, more tailored studies using modern models and analysis techniques are required that reproduce the observed spectra and other indicators simultaneously. In view of this, only four other He-strong stars analysed in analogy to here (Przybilla et al. 2016; Castro et al. 2017; González et al. 2017, 2019) remain for a comparison, such that no firm conclusions can be drawn on the reason for HD144941's extreme helium enrichment. We may speculate that because of the lower $T_{\text {eff }}$ (less radiation pressure), its higher surface gravity, and much stronger magnetic field, the He fallback is more efficient and a large chemically peculiar cap is formed around the magnetic pole that dominates the visible hemisphere (see Sect. 3).

The stellar radius can be then determined from stellar mass and surface gravity. Together with the rotation period from the $\mathrm{K} 2$ light curve, the equatorial rotational velocity is determined as $\sim 14 \mathrm{~km} \mathrm{~s}^{-1}$. Our constraint on $v \sin i$ is not very accurate because of the degeneracy with macroturbulence. As a consequence, the inclination angle $i$ between the rotational axis and line of sight is only loosely constrained to values between $\sim 9$ and $60^{\circ}(1 \sigma$ - 


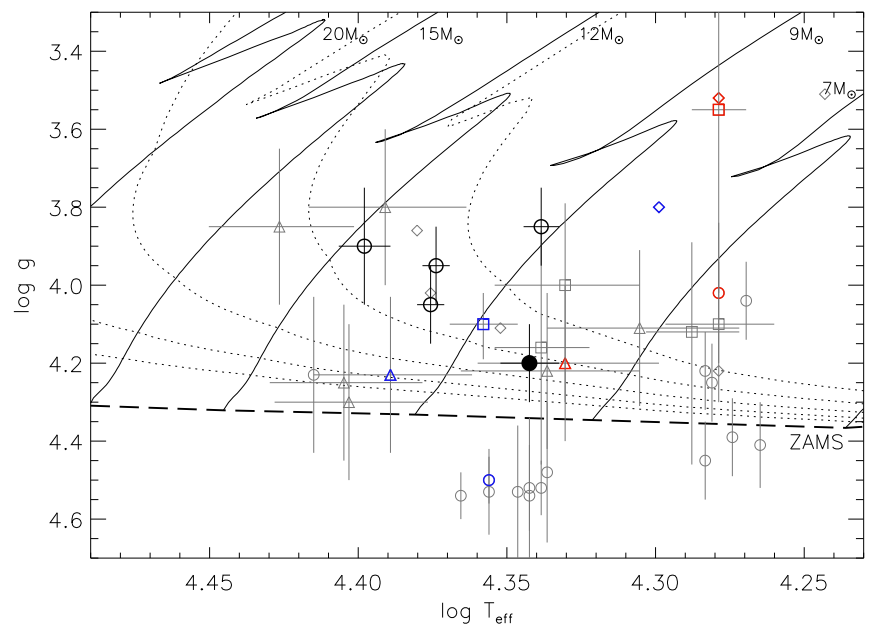

Fig. 7. Kiel diagram for HD144941 (black dot). Geneva evolution tracks for rotating stars are shown for metallicity $Z=0.014$ and an initial value of $40 \%$ of the critical rotation rate (Ekström et al. 2012 full lines). Corresponding isochrones for $\log \tau(\mathrm{Myr})=6.5$ to 7.3 , with 0.2 spacing are also indicated (dotted lines, bottom to top), as well as the position of the zero-age main sequence (long dashed line). Positions of other He-strong stars analysed in analogy to the present work are shown (open black circles). Literature values for He-strong stars are marked by grey symbols: from Zboril et al. (1997 circles), Leone et al. (1997 diamonds), Hunger \& Groote (1999 squares) and Cidale et al. (2007 triangles). Two stars common to all four literature analyses are highlighted, HD58260 (red symbols) and HD64740 (blue symbols). Where available, $1 \sigma$-error bars are displayed.

range). The luminosity can finally be determined from $R$ and $T_{\text {eff }}$, which is in excellent agreement with $M_{\text {bol }}$ determined previously. The age and the main-sequence lifetime are deduced from the evolutionary tracks. All fundamental parameter data are incorporated in Table 2.

\section{Concluding remarks}

Finally, we resume the discussion of the slightly discrepant fits to some of the observed spectral lines, the smaller model widths of the red $\mathrm{He}_{\mathrm{I}}$ lines than observed, and the mismatch of the fits to the cores of some metal lines (while others appear to be fitted fine). The key to understanding lies in the $C_{\text {II }} \lambda \lambda 6578 / 82 \AA$ multiplet. Both lines show a pronounced 'W'-shaped profile, which is also consistently seen in other strong metal lines, although often barely above the noise level (see Fig. 8). The discrepancies in the widths of the He I line cores in the red can also be largely resolved by assuming them to stem from Zeeman splitting. We model the Zeeman splitting in our line-formation calculations by applying shifts and relative component strengths as described by Sobelman (1992). A good fit is obtained in Fig. 8 for a field strength of $15 \mathrm{kG}$ by assuming a pure longitudinal field configuration; only the $\sigma$-components are visible and the $\pi$-components are essentially absent from the observations of the metal lines. In addition to the spectropolarimetry, this puts an independent constraint on the minimum magnetic field strength. The Zeeman splitting results in an extra line broadening $\propto \lambda^{2}$ (as observed in the red $\mathrm{He}_{\mathrm{I}}$ lines), which for most lines is not resolved and can be accounted for by an increase in the macroturbulent velocity. Elemental abundances are not significantly affected for most of the features because the metal lines are very weak and formed on the linear part of the curve of growth; that is, the splitting does

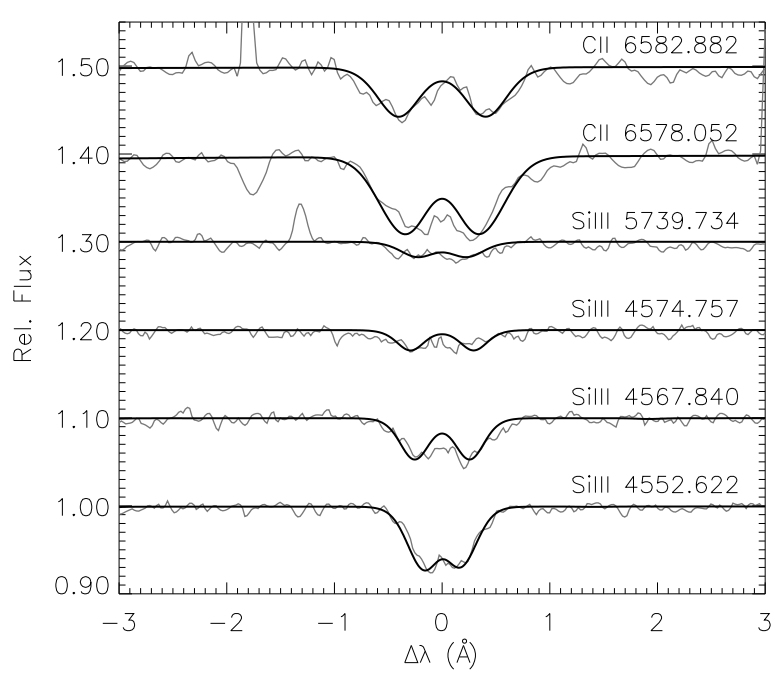

Fig. 8. Modelling (black line) of the Zeeman splitting assuming a longitudinal field of $15 \mathrm{kG}$ for several observed spectral features (grey lines). Displayed are relative fluxes, each offset by 0.1 units, as a function of wavelength difference relative to the line centre. A line identification and the laboratory wavelengths are indicated for each feature.

not lead to de-saturation. For the case where we account for the Zeeman splitting, $\zeta$ can be reduced from 25 to $15 \mathrm{~km} \mathrm{~s}^{-1}$.

A parallel work on HD144941, Shultz et al. (2021) find very similar magnetic properties for the star from high-resolution spectropolarimetry and spectroscopy. Moreover, a detection of weak $\mathrm{H} \alpha$ emission is reported for the first time, suggesting the existence of a centrifugal magnetosphere (Petit et al. 2013). Using the rigidly rotating magnetosphere model of Townsend \& Owocki (2005), they propose this as strong evidence of the star having a mass around $1 M_{\odot}$, which they derive from adopting the radius determined from the Gaia EDR3 parallax and the low surface gravity value of Pandey \& Lambert (2017) that we ruled out here. Interestingly, Shultz et al. (2021) note that their model could be reconciled with the massive star nature of HD144941 in important aspects if $v \sin i$ were lower than what they derived $\left(11.2 \pm 0.7 \mathrm{~km} \mathrm{~s}^{-1}\right.$ ), requiring about $7 \mathrm{~km} \mathrm{~s}^{-1}$ (c.f. our value in Table 2, though unfortunately with a large uncertainty). They recognise their sparse rotational phase coverage as a potential caveat in their discussion, such that we remain optimistic that further observations will allow a fully consistent picture to be derived.

We conclude that all available observational constraints investigated here imply that HD144941 is the most extreme member of the class of He-strong stars (main-sequence B stars where atmospheric hydrogen and metals have been substantially depleted by a fractionated stellar wind), and that it not a member of the EHe-class of stars (low-mass stellar remnants with atmospheres comprising nuclear ash from CNO-cycle hydrogen burning and $3 \alpha$ helium burning). The star HD144941 is unique with regard to helium content in the visible hemisphere, with almost all of the atmospheric hydrogen substituted by helium. Follow-up time-series spectropolarimetry at high spectral resolution would be desirable to investigate the magnetosphere in depth and to study the detailed properties of the magnetic field geometry and spotted distribution of chemical elements on the star's surface via Zeeman Doppler tomography.

Acknowledgements. Based on observations collected at the European Southern Observatory under ESO programmes 077.D-0458(A,B), 105.205U.001 and 106.211U.001. We are grateful to K. Butler for valuable discussion and providing and maintaining DetaIL/Surface, and to A. Irrgang for updates of the codes. 


\section{References}

Allen, C. \& Santillan, A. 1991, Rev. Mexicana Astron. Astrofis., 22, 255 Angel, J. R. P. \& Landstreet, J. D. 1970, ApJ, 160, L147

Appenzeller, I., Fricke, K., Fürtig, W., et al. 1998, The Messenger, 94, 1

Asplund, M., Grevesse, N., Sauval, A. J., \& Scott, P. 2009, ARA\&A, 47, 481

Bagnulo, S., Fossati, L., Kochukhov, O., \& Landstreet, J. D. 2013, A\&A, 559, A103

Bagnulo, S., Fossati, L., Landstreet, J. D., \& Izzo, C. 2015, A\&A, 583, A115

Bagnulo, S., Landstreet, J. D., Fossati, L., \& Kochukhov, O. 2012, A\&A, 538, A 129

Bagnulo, S., Szeifert, T., Wade, G. A., Landstreet, J. D., \& Mathys, G. 2002, A\&A, 389, 191

Beauchamp, A., Wesemael, F., \& Bergeron, P. 1997, ApJS, 108, 559

Borra, E. F. \& Landstreet, J. D. 1980, ApJS, 42, 421

Butler, K. \& Giddings, J. R. 1985, Newsletter of Analysis of Astronomical Spectra, 9 (Univ. London)

Cardelli, J. A., Clayton, G. C., \& Mathis, J. S. 1989, ApJ, 345, 245

Castro, N., Fossati, L., Hubrig, S., et al. 2017, A\&A, 597, L6

Cidale, L. S., Arias, M. L., Torres, A. F., et al. 2007, A\&A, 468, 263

Cutri, R. M., Wright, E. L., Conrow, T., et al. 2021, VizieR Online Data Catalog, II/328

Dekker, H., D’Odorico, S., Kaufer, A., et al. 2000, Proc. SPIE, 4008, 534

Ekström, S., Georgy, C., Eggenberger, P., et al. 2012, A\&A, 537, A146

Fossati, L., Castro, N., Schöller, M., et al. 2015, A\&A, 582, A45

Froese Fischer, C. \& Tachiev, G. 2004, At. Data Nucl. Data Tables, 87, 1

Froese Fischer, C., Tachiev, G., \& Irimia, A. 2006, At. Data Nucl. Data Tables, 92,607

Fuhr, J. R. \& Wiese, W. L. 1998, in CRC Handbook of Chemistry and Physics, 79th edn., ed. D. R. Lide (Boca Raton: CRC Press)

Gaia Collaboration, Brown, A. G. A., Vallenari, A., et al. 2021, A\&A, 649, A1

Gaia Collaboration, Prusti, T., de Bruijne, J. H. J., et al. 2016, A\&A, 595, A1

Ghazaryan, S., Alecian, G., \& Hakobyan, A. A. 2019, MNRAS, 487, 5922

Giddings, J. R. 1981, PhD thesis, (Univ. London)

González, J. F., Briquet, M., Przybilla, N., et al. 2019, A\&A, 626, A94

González, J. F., Hubrig, S., Przybilla, N., et al. 2017, MNRAS, 467, 437

Harrison, P. M. \& Jeffery, C. S. 1997, A\&A, 323, 177

Hunger, K. \& Groote, D. 1999, A\&A, 351, 554

Hunger, K. \& Kaufmann, J. P. 1973, A\&A, 25, 261

Jeffery, C. S. 2008, ASP Conf. Ser., 391, 53

Jeffery, C. S. \& Harrison, P. M. 1997, A\&A, 323, 393

Jeffery, C. S. \& Ramsay, G. 2018, MNRAS, 475, L122

Kaufer, A., Stahl, O., Tubbesing, S., et al. 1999, The Messenger, 95, 8

Kelleher, D. E. \& Podobedova, L. I. 2008, J. Phys. \& Chem. Ref. Data, 37, 1285

Koester, D., Napiwotzki, R., Christlieb, N., et al. 2001, A\&A, 378, 556

Kupfer, T., Przybilla, N., Heber, U., et al. 2017, MNRAS, 471, 877

Kurucz, R. \& Bell, B. 1995, CD-ROM No. 23 (Cambridge, Mass: SAO)

Kurucz, R. L. 1996, ASP Conf. Ser., 108, 160

Landstreet, J. D., Bagnulo, S., \& Fossati, L. 2014, A\&A, 572, A113

Leone, F., Catalano, F. A., \& Malaroda, S. 1997, A\&A, 325, 1125

MacConnell, D. J., Frye, R. L., \& Bidelman, W. P. 1970, PASP, 82, 730

Mar, S., Pérez, C., González, V. R., et al. 2000, A\&AS, 144, 509

Nieva, M. F. \& Przybilla, N. 2007, A\&A, 467, 295

Nieva, M.-F. \& Przybilla, N. 2012, A\&A, 539, A143

Nieva, M.-F. \& Przybilla, N. 2014, A\&A, 566, A7

Odenkirchen, M. \& Brosche, P. 1992, Astronomische Nachrichten, 313, 69

Pandey, G. \& Lambert, D. L. 2017, ApJ, 847, 127

Pauli, E. M., Napiwotzki, R., Heber, U., et al. 2006, A\&A, 447, 173

Petit, V., Owocki, S. P., Wade, G. A., et al. 2013, MNRAS, 429, 398

Przybilla, N. 2005, A\&A, 443, 293

Przybilla, N. \& Butler, K. 2004, ApJ, 609, 1181

Przybilla, N., Butler, K., Heber, U., \& Jeffery, C. S. 2005, A\&A, 443, L25

Przybilla, N., Fossati, L., Hubrig, S., et al. 2016, A\&A, 587, A7

Przybilla, N., Nieva, M.-F., \& Butler, K. 2011, J. Phys. Conf. Ser., 328, 012015

Przybilla, N., Nieva, M. F., Heber, U., \& Jeffery, C. S. 2006, Balt. Astron., 15, 163

Przybilla, N., Nieva, M. F., Irrgang, A., \& Butler, K. 2013, EAS Publ. Ser., 63, 13

Ren, F., Chen, X., Zhang, H., et al. 2021, ApJ, 911, L20

Saio, H. \& Jeffery, C. S. 2000, MNRAS, 313, 671

Shultz, M. E., Kochukhov, O., Labadie-Bartz, J., David-Uraz, A., \& Owocki, S. P. 2021, arXiv e-prints, arXiv:2107.11211

Skrutskie, M. F., Cutri, R. M., Stiening, R., et al. 2006, AJ, 131, 1163

Slawson, R. W., Hill, R. J., \& Landstreet, J. D. 1992, ApJS, 82, 117

Smith, K. C. 1996, Ap\&SS, 237, 77

Sobelman, I. I. 1992, Atomic Spectra and Radiative Transitions, 2nd edition (Springer, Berlin Heidelberg)

Townsend, R. H. D. \& Owocki, S. P. 2005, MNRAS, 357, 251

Tremblay, P. E. \& Bergeron, P. 2009, ApJ, 696, 1755

Wiese, W. L. \& Fuhr, J. R. 2009, J. Phys. \& Chem. Ref. Data, 38, 565

Wiese, W. L., Fuhr, J. R., \& Deters, T. M. 1996, J. Phys. \& Chem. Ref. Data, Mon., 7

Wiese, W. L., Smith, M. W., \& Miles, B. M. 1969, Nat. Stand. Ref. Data Ser.,

Nat. Bur. Stand. (U.S.), NSRDS-NBS 22, Vol. II

Zboril, M., North, P., Glagolevskij, Y. V., \& Betrix, F. 1997, A\&A, 324, 949 


\section{Appendix A: Line abundances}

Table A.1. Line-by-line abundances from the non-LTE analysis of HD144941. Abundances $\log n_{X}$ (normalised to $\sum n_{X}=1$ ).

\begin{tabular}{|c|c|c|c|c|c|c|}
\hline Line & $\lambda(\AA)$ & $\chi(\mathrm{eV})$ & $\log g f$ & Accu. & Source & $\log n_{X}$ \\
\hline $\mathrm{H} \delta$ & 4101.742 & 10.20 & -0.75243 & AAA & WF & -1.30 \\
\hline $\mathrm{H} \gamma$ & 4340.471 & 10.20 & -0.44666 & AAA & WF & -1.28 \\
\hline $\mathrm{H} \beta$ & 4861.333 & 10.20 & -0.01996 & AAA & WF & -1.33 \\
\hline $\mathrm{H} \alpha$ & 6562.819 & 10.20 & 0.71000 & AAA & WF & -1.30 \\
\hline $\mathrm{C}_{\text {II }}$ & 3918.968 & 16.33 & -0.533 & B & WFD & -4.75 \\
\hline $\mathrm{C}_{\text {II }}$ & 3920.681 & 16.33 & -0.232 & B & WFD & -4.62 \\
\hline $\mathrm{C}_{\text {II }}$ & 4267.001 & 18.05 & 0.562 & $\mathrm{C}+$ & WFD & -4.83 \\
\hline $\mathrm{C}_{\text {II }}$ & 4267.261 & 18.05 & 0.717 & $\mathrm{C}+$ & WFD & \\
\hline $\mathrm{C}_{\text {II }}$ & 4267.261 & 18.05 & -0.584 & $\mathrm{C}+$ & WFD & \\
\hline $\mathrm{C}_{\text {II }}$ & 5132.947 & 20.70 & -0.211 & B & WFD & -4.52 \\
\hline $\mathrm{C}_{\text {II }}$ & 5133.282 & 20.70 & -0.178 & B & WFD & \\
\hline $\mathrm{C}_{\text {II }}$ & 5143.495 & 20.70 & -0.212 & B & WFD & -4.54 \\
\hline $\mathrm{C}_{\text {II }}$ & 5145.165 & 20.71 & 0.189 & B & WFD & -4.61 \\
\hline $\mathrm{C}_{\text {II }}$ & 5151.085 & 20.71 & -0.179 & B & WFD & -4.54 \\
\hline $\mathrm{N}_{\text {II }}$ & 3994.997 & 18.50 & 0.163 & B & FFT & -5.11 \\
\hline $\mathrm{N}_{\text {II }}$ & 4041.310 & 23.14 & 0.748 & B & MAR & -4.91 \\
\hline $\mathrm{N}_{\text {II }}$ & 4236.927 & 23.24 & 0.410 & $X$ & K14 & -5.10 \\
\hline $\mathrm{N}_{\text {II }}$ & 4237.047 & 23.24 & 0.319 & $X$ & K14 & \\
\hline $\mathrm{N}_{\text {II }}$ & 4241.755 & 23.24 & 0.142 & $X$ & K14 & -5.12 \\
\hline $\mathrm{N}_{\text {II }}$ & 4241.786 & 23.24 & 0.657 & $X$ & K14 & \\
\hline $\mathrm{N}_{\text {II }}$ & 4447.030 & 20.41 & 0.221 & B & FFT & -4.99 \\
\hline $\mathrm{N}_{\text {II }}$ & 4601.478 & 18.47 & -0.452 & $B+$ & FFT & -5.00 \\
\hline $\mathrm{N}_{\text {II }}$ & 4607.153 & 18.46 & -0.522 & $\mathrm{~B}+$ & FFT & -5.09 \\
\hline $\mathrm{N}_{\text {II }}$ & 4621.393 & 18.47 & -0.538 & $B+$ & FFT & -5.08 \\
\hline $\mathrm{N}_{\text {II }}$ & 4630.539 & 18.48 & 0.080 & $\mathrm{~B}+$ & FFT & -5.13 \\
\hline $\mathrm{N}_{\text {II }}$ & 4643.086 & 18.48 & -0.371 & $\mathrm{~B}+$ & FFT & -5.07 \\
\hline $\mathrm{N}_{\text {II }}$ & 5001.474 & 20.65 & 0.435 & B & FFT & -5.07 \\
\hline $\mathrm{N}_{\text {II }}$ & 5005. & 20.67 & 0.587 & B & FFT & -4.91 \\
\hline $\mathrm{N}_{\text {II }}$ & 5666.629 & 18.47 & -0.104 & $\mathrm{~B}+$ & MAR & -4.99 \\
\hline $\mathrm{N}_{\text {II }}$ & 5676.017 & 18.46 & -0.356 & $\mathrm{~B}+$ & MAR & -5.02 \\
\hline $\mathrm{N}_{\text {II }}$ & 5679.558 & 18.48 & 0.221 & $\mathrm{~B}+$ & MAR & -5.18 \\
\hline $\mathrm{O}_{\mathrm{I}}$ & 7771.944 & 9.15 & 0.354 & A & FFT & -4.20 \\
\hline $\mathrm{O}_{\text {II }}$ & 3945.038 & 23.42 & -0.711 & $\mathrm{~B}+$ & FFT & -4.12 \\
\hline $\mathrm{O}_{\text {II }}$ & 3954.362 & 23.42 & -0.402 & $B+$ & FFT & -4.27 \\
\hline $\mathrm{O}_{\text {II }}$ & 4069.623 & 25.63 & 0.144 & $\mathrm{~B}+$ & FFT & -4.33 \\
\hline $\mathrm{O}_{\text {II }}$ & 4069.882 & 25.64 & 0.352 & $B+$ & FFT & \\
\hline $\mathrm{O}_{\text {II }}$ & 4072.153 & 25.65 & 0.528 & $\mathrm{~B}+$ & FFT & -4.33 \\
\hline $\mathrm{O}_{\text {II }}$ & 4075.862 & 25.67 & 693 & $\mathrm{~B}+$ & FFT & -4.13 \\
\hline $\mathrm{O}_{\text {II }}$ & 4085.112 & 25.65 & -0.191 & $\mathrm{~B}+$ & FFT & -4.24 \\
\hline $\mathrm{O}_{\text {II }}$ & 415 & 25. & 070 & $\mathrm{~B}+$ & FFT & -4.15 \\
\hline $\mathrm{O}_{\text {II }}$ & 4317.139 & 22.97 & -0.368 & $\mathrm{~B}+$ & FFT & -4.25 \\
\hline $\mathrm{O}_{\text {II }}$ & 4319.630 & 22.98 & -0.372 & $\mathrm{~B}+$ & FFT & -4.32 \\
\hline $\mathrm{O}_{\text {II }}$ & 4345.560 & 22.98 & -0.342 & $\mathrm{~B}+$ & FFT & -4.33 \\
\hline $\mathrm{O}_{\text {II }}$ & 4349.426 & 23.00 & 0.073 & $\mathrm{~B}+$ & FFT & -4.38 \\
\hline $\mathrm{O}_{\text {II }}$ & 4351.260 & 25.66 & 0.202 & $\mathrm{~B}+$ & FFT & -4.32 \\
\hline $\mathrm{O}_{\text {II }}$ & 4351.457 & 25.66 & -1.013 & B & FFT & \\
\hline $\mathrm{O}_{\text {II }}$ & 4366.892 & 23.00 & -0.333 & $\mathrm{~B}+$ & FFT & -4.31 \\
\hline $\mathrm{O}_{\text {II }}$ & 4414.905 & 23.44 & 0.207 & B & FFT & -4.26 \\
\hline $\mathrm{O}_{\text {II }}$ & 4416.974 & 23.42 & -0.043 & B & FFT & -4.22 \\
\hline $\mathrm{O}_{\text {II }}$ & 4590.974 & 25.66 & 0.331 & $B+$ & FFT & -4.28 \\
\hline $\mathrm{O}_{\text {II }}$ & 4595.957 & 25.66 & -1.022 & B & FFT & -4.27 \\
\hline $\mathrm{O}_{\text {II }}$ & 4596.175 & 25.66 & 0.180 & $\mathrm{~B}+$ & FFT & \\
\hline $\mathrm{O}_{\text {II }}$ & 4638.856 & 22.97 & -0.324 & $B+$ & FFT & -4.21 \\
\hline $\mathrm{O}_{\text {II }}$ & 4641.810 & 22.98 & 0.066 & $\mathrm{~B}+$ & FFT & -4.30 \\
\hline $\mathrm{O}_{\text {II }}$ & 4649.135 & 23.00 & 0.324 & $\mathrm{~B}+$ & FFT & -4.31 \\
\hline $\mathrm{O}_{\text {II }}$ & 4650.839 & 22.97 & -0.349 & $\mathrm{~B}+$ & FFT & -4.25 \\
\hline $\mathrm{Ne}_{I}$ & 6143.063 & 16.62 & -0.070 & $\mathrm{~B}+$ & FFT & -4.70 \\
\hline $\mathrm{Ne}_{I}$ & 6402.248 & 16.62 & 0.365 & $\mathrm{~B}+$ & FFT & -4.65 \\
\hline
\end{tabular}

Table A.1. (cont.)

\begin{tabular}{|c|c|c|c|c|c|c|}
\hline Line & $\lambda(\AA)$ & $\chi(\mathrm{eV})$ & $\log g f$ & Accu. & Source & $\log n_{X}$ \\
\hline $\mathrm{Mg}_{\text {II }}$ & 4481.126 & 8.86 & 0.730 & $\mathrm{~B}$ & FW & -5.52 \\
\hline $\mathrm{Mg}_{\text {II }}$ & 4481.150 & 8.86 & -0.570 & B & FW & \\
\hline $\mathrm{Mg}_{\text {II }}$ & 4481.325 & 8.86 & 0.575 & B & FW & \\
\hline $\mathrm{Al}$ III & 4512.565 & 17.81 & 0.408 & $\mathrm{~A}+$ & FFTI & -6.57 \\
\hline $\mathrm{A} l_{\text {III }}$ & 4528.945 & 17.82 & -0.291 & $\mathrm{~A}+$ & FFTI & -6.67 \\
\hline $\mathrm{Al}$ III & 4529.189 & 17.82 & 0.663 & $\mathrm{~A}+$ & FFTI & -6.71 \\
\hline $\mathrm{Al}$ III & 5696.604 & 15.64 & 0.232 & $\mathrm{~A}+$ & FFTI & -6.72 \\
\hline $\mathrm{Si}$ II & 3856.018 & 6.86 & -0.442 & $\mathrm{C}+$ & FFTI & -5.59 \\
\hline $\mathrm{Si}$ II & 3862.595 & 6.86 & -0.757 & $\mathrm{C}+$ & KP & -5.32 \\
\hline Si II & 4128.054 & 9.84 & 0.359 & B & KP & -5.46 \\
\hline Si II & 4130.872 & 9.84 & -0.783 & $\mathrm{D}+$ & KP & -5.57 \\
\hline Si II & 4130.894 & 9.84 & 0.552 & B & KP & \\
\hline Si II & 5041.024 & 10.07 & 0.17 & $\mathrm{D}+$ & WSM & -5.53 \\
\hline $\mathrm{Si}$ II & 5055.984 & 10.07 & 0.42 & $\mathrm{D}+$ & WSM & -5.62 \\
\hline Si II & 5056.317 & 10.07 & -0.53 & E & WSM & \\
\hline Si II & 6347.109 & 8.12 & 0.177 & $\mathrm{~B}+$ & FFTI & -5.34 \\
\hline Si II & 6371.371 & 8.12 & -0.126 & $\mathrm{C}+$ & FFTI & -5.43 \\
\hline Si III & 4552.622 & 19.02 & 0.292 & $\mathrm{C}+$ & FFTI & -5.57 \\
\hline Si III & 4567.840 & 19.02 & 0.068 & $\mathrm{~B}+$ & FFTI & -5.57 \\
\hline Si III & 4574.757 & 19.02 & -0.409 & B & FFTI & -5.64 \\
\hline Si III & 4813.333 & 25.98 & 0.708 & $\mathrm{~B}+$ & KP & -5.42 \\
\hline Si III & 4819.631 & 25.98 & -1.841 & $\mathrm{X}$ & K13 & -5.38 \\
\hline Si III & 4819.712 & 25.98 & 0.823 & $\mathrm{~B}+$ & KP & \\
\hline Si III & 4819.814 & 25.98 & -0.353 & B & KP & \\
\hline Si III & 4828.950 & 25.99 & 0.937 & $\mathrm{~B}+$ & KP & -5.38 \\
\hline Si III & 4829.029 & 25.99 & -3.169 & $X$ & K13 & \\
\hline Si III & 4829.111 & 25.99 & -0.353 & B & KP & \\
\hline Si III & 4829.214 & 25.99 & -2.170 & $\mathrm{D}+$ & KP & \\
\hline Si III & 5739.734 & 19.72 & -0.078 & B & FFTI & -5.40 \\
\hline $\mathrm{S}_{\text {II }}$ & 4162.665 & 15.94 & 0.78 & $\mathrm{D}$ & WSM & -5.67 \\
\hline$S_{\text {II }}$ & 5432.797 & 13.62 & 0.205 & $\mathrm{C}+$ & FFTI & -5.54 \\
\hline$S_{\text {II }}$ & 5453.855 & 13.67 & 0.442 & $\mathrm{C}+$ & FFTI & -5.52 \\
\hline$S_{\text {III }}$ & 4253.499 & 18.24 & 0.107 & $\mathrm{~B}+$ & FFTI & -5.74 \\
\hline$S_{\text {III }}$ & 4284.904 & 18.19 & -0.233 & $\mathrm{~B}+$ & FFTI & -5.54 \\
\hline Fe III & 4164.731 & 20.63 & 0.923 & X & $\mathrm{KB}$ & -5.54 \\
\hline $\mathrm{Fe}$ III & 4164.916 & 24.65 & 1.011 & $\mathrm{X}$ & $\mathrm{KB}$ & \\
\hline Fe III & 4372.039 & 22.91 & 0.585 & $\mathrm{X}$ & KB & -5.36 \\
\hline Fe III & 4372.096 & 22.91 & 0.029 & $\mathrm{X}$ & KB & \\
\hline $\mathrm{Fe}$ III & 4372.134 & 22.91 & 0.727 & $X$ & KB & \\
\hline $\mathrm{Fe}_{\text {III }}$ & 4372.306 & 22.91 & 0.865 & $X$ & KB & \\
\hline $\mathrm{Fe}_{\text {III }}$ & 4372.306 & 22.91 & 0.193 & $\mathrm{X}$ & $\mathrm{KB}$ & \\
\hline $\mathrm{Fe}$ III & 4372.497 & 22.91 & 0.200 & $\mathrm{X}$ & KB & \\
\hline $\mathrm{Fe}$ III & 4372.536 & 22.91 & 0.993 & X & KB & \\
\hline $\mathrm{Fe}$ III & 4372.784 & 22.91 & 0.040 & $\mathrm{X}$ & KB & \\
\hline $\mathrm{Fe}$ III & 4372.823 & 22.91 & 1.112 & $X$ & KB & \\
\hline $\mathrm{Fe}$ III & 4419.596 & 8.24 & -2.218 & $X$ & KB & -5.39 \\
\hline $\mathrm{Fe}$ III & 5127.387 & 8.66 & -2.218 & $\mathrm{X}$ & KB & -5.22 \\
\hline $\mathrm{Fe}$ III & 5127.631 & 8.66 & -2.564 & $X$ & KB & \\
\hline $\mathrm{Fe}_{\text {III }}$ & 5156.111 & 8.64 & -2.018 & $X$ & KB & -5.53 \\
\hline
\end{tabular}

Notes. Accuracy indicators - uncertainties within the following: AAA: $0.3 \%$; AA: $1 \%$; A: $3 \%$; B: $10 \%$; C: $25 \%$; D: $50 \%$; E: $>50 \%$; X: unknown. Sources of $g f$-values: FFT: Froese Fischer \& Tachiev (2004); FFTI: Froese Fischer et al. (2006); FW: Fuhr \& Wiese (1998); K13, K14: http://kurucz.harvard.edu/atoms.html; KB: Kurucz \& Bell (1995); KP: Kelleher \& Podobedova (2008); MAR: Mar et al. (2000); WF: Wiese \& Fuhr (2009); WFD: Wiese et al. (1996); WSM: Wiese et al. (1969). 